\title{
Globalisierung und europäische Sozialpolitik. Implikationen der Strategie „Europa 2020“
}

von Thilo Fehmel

Von der politischen Öffentlichkeit weitgehend unbeachtet, beerdigte die Europäische Kommission die hochfliegenden Ziele der im Jahr 2000 verabschiedeten LissabonStrategie und legte sich in der 2010 vorgestellten Nachfolgestrategie ,Europa 2020 “ auf deutlich nüchternere Ziele fest. Insbesondere wird mit dem Entwurf einer „Innovationsunion " der wachsenden globalen Bedeutung nicht-europäischer Wirtschaftsregionen Rechnung getragen. Der Beitrag widmet sich in zwei Schritten den sozialpolitischen Erfolgsbedingungen dieser ,Innovationsunion“. Zunächst wird grundsätzlich herausgearbeitet, inwiefern Sozialstaatlichkeit als Besonderheit europäischer Ökonomien zu deren Erfolg im globalen Wettbewerb beiträgt. Dann wird - ausgehend von soziologischen Befunden zum Verhältnis von Innovation und Sicherheit - untersucht, welche Form von Sozialpolitik dem ökonomischen Anspruch der ,Innovationsunion“ am besten gerecht wird.

Largely ignored by the general public, the European Commission has scrapped the Lisbon Strategy of the year 2000 and subsequently proclaimed new and far more realistic objectives in its follow-up strategy "Europe 2020", presented in 2010. In particular, the increasing relevance of non-European economic regions is taken into account through the concept of an "Innovation Union". The article examines the socio-political conditions of success for such an "Innovation Union" in two steps. First, it is shown how the welfare state, as a distinctive feature of European economies, contributes to their success in a globally competitive environment. Second, based on sociological data on the relationship between innovation and security, the particular type of social policy that best fits the economic demands of the "Innovation Union" is outlined.

\section{Einleitung}

Die innereuropäischen Probleme der letzten Jahre haben die Frage nach der wirtschaftlichen Bedeutung Europas in der Welt in den Hintergrund gedrängt. Auch einem zentralen Zukunftsentwurf der Europäischen Kommission widerfuhr das Schicksal mangelnder Aufmerksamkeit: dem Strategiepapier „Europa 2020“. ${ }^{\text {Es }}$

1 Europäische Kommission: Europa 2020 - Eine Strategie für intelligentes, nachhaltiges und integratives Wachstum. Mitteilung der Kommission, Brüssel (KOM(2010) 2020 endg.), 2010. 
gilt als Nachfolger der - ungleich bekannteren - Lissabon-Strategie, fällt im Vergleich zu dieser jedoch bodenständiger und deutlich weniger ambitioniert aus. Vor allem basieren seine Ausführungen auf realistischeren Einschätzungen zur Stellung Europas im globalen wirtschaftlichen Wettbewerb. Die Europäische Kommission verbindet diese Selbstrelativierung mit dem Entwurf einer „Innovationsunion" und erwähnt explizit die damit verbundenen sozialpolitischen Herausforderungen. Diese Verknüpfung gibt den Anstoß zum vorliegenden Beitrag. Er widmet sich den sozialpolitischen Erfolgsbedingungen der ausgerufenen „Innovationsunion“. Es wird gezeigt, welchen Beitrag Sozialstaatlichkeit als Besonderheit europäischer Volkswirtschaften zu deren Erfolg im globalen Wettbewerb leistet, und es wird dargestellt, welche konkrete Form von Sozialpolitik hierfür am geeignetsten ist.

Aufbauend auf einer verbreiteten Unterscheidung möglicher Arenen der Wohlfahrtsproduktion (Familie/Gemeinschaft, Markt, Staat ${ }^{2}$ ) werden Nordamerika, Ostasien und Europa als differente Wirtschaftssysteme mit differenten Hierarchien der Wohlfahrtsarenen beschrieben (II.). Diese Differenzierung macht es möglich, Diskurse zum Verhältnis von ökonomischer Globalisierung und Sozialpolitik als europäische Besonderheit zu markieren und im Weiteren zwischen diesbezüglich pessimistischen und optimistischen Auffassungen zu unterscheiden (III.). Ausweislich der expliziten Verknüpfung von Innovation und sozialer Sicherheit ist der Europäischen Kommission eine grundsätzlich optimistische Sichtweise auf die Möglichkeiten sozialpolitischer Flankierung der ökonomischen Globalisierung zu attestieren. Vor welchen Herausforderungen sie dabei steht, wird unter IV. anhand soziologischer Befunde zu den Themen Innovationsfähigkeit (Bildung) und Innovationsbereitschaft (Unsicherheit) illustriert. Unter V. wird auf dieser Basis der Blick geschärft für notwendige Merkmale, über die Systeme sozialer Sicherung in Europa verfügen sollten, wenn sie den Ansprüchen der „Innovationsunion“" genügen sollen. Der Beitrag schließt mit einer Diskussion der nicht-intendierten emanzipatorischen Potenziale einer solchen dem Grunde nach auf employability - ausgerichteten Art und Weise sozialer Sicherung.

2 Esping-Andersen, G.: The three worlds of welfare capitalism, Princeton (N.J.), 1990. 


\section{II. Ökonomische Globalisierung}

Das, was seit einiger Zeit als Globalisierung diskutiert wird, ist kein neuartiges Phänomen. ${ }^{3}$ Es ist nicht einmal ein Alleinstellungsmerkmal der Neuzeit. Transimperiale und transnationale, darunter auch interkontinentale Bewegungen, Beziehungen und Abhängigkeiten sind seit Jahrtausenden nachweisbar. Aber sie sind in der Regel keine kontinuierlich ablaufenden Prozesse. Da sie sich in Wellen und Schüben vollzogen und vollziehen, werden sie auch in Wellen und Schüben wahrgenommen. Daraus ergibt sich die „Chance“ der jeweiligen Beobachter, den aktuellen Schub als exzeptionell zu bewerten.

Das gilt auch für die Beobachtungen und Deutungen jener Prozesse institutionellen Wandels, aus denen sich in den letzten Dekaden wechselseitige Beeinflussungen und intensivierte Abhängigkeiten räumlich weit entfernter Lebensverhältnisse ergeben haben. ${ }^{4}$ Diesen Beobachtungen zufolge sind die Sozialsysteme Europas insbesondere von zwei Entwicklungen betroffen, die mit dem Begriff der Globalisierung in Verbindung gebracht werden, zumindest vordergründig aber wenig miteinander zu tun haben. Im Folgenden werden die beiden Entwicklungen, um sie voneinander unterscheiden zu können, als horizontale und als vertikale Globalisierung bezeichnet, unter grober Orientierung an den jeweiligen Stromrichtungen. Die grenzüberschreitende Bewegung von Wirtschaftsgütern (Handel) und Kapital vollzieht sich ganz überwiegend horizontal, und hier wiederum nahezu ausschließlich zwischen clustern der nördlichen Hemisphäre. Involviert sind Länder und Agglomerationen mit hoher wirtschaftlicher Produktivität und umfangreichen finanziellen Ressourcen, also Nordamerika, Europa, Nordost-Asien inkl. China. Die grenzüberschreitende Bewegung von Personen (Migration) vollzieht sich dagegen ganz überwiegend in vertikaler Richtung, zwischen den armen Gesellschaften des Südens und den jeweils darüber liegenden wohlhabenden Wirtschaftsclustern des Nordens.

Waren- und Kapital- sowie Migrationsströme bewegen sich also weder gleichgerichtet noch jeweils global. Angemessener als die Rede von Globalisierung wäre daher die von zunehmender ökonomischer (horizontaler) und sozialer (vertikaler) regionaler Transnationalisierung. Allerdings wird der Begriff hier bewusst beibehalten, da dies auch jene relevanten Akteure tun, deren Problemdeutungen und

3 Hirst, P./Thompson, G.: Globalization in Question, Cambridge, 1996.

4 Vobruba, G.: Globalisierung und ihre Interpretationen. Soziologie der Transnationalisierung als Beobachtung zweiter Ordnung., in: Nissen, S./Vobruba, G. (Hg.): Die Ökonomie der Gesellschaft, Wiesbaden, 2009, 225-239, 228f. 
Lösungsfavoriten die Grundlage der nachfolgenden Ausführungen sind. So wird also zum einen (unter III.) der Globalisierungsbegriff genutzt, da er im einschlägigen öffentlichen und akademischen Diskurs breit eingeführt ist. Zum anderen ist (unter IV.) von Globalisierung die Rede, weil dies der Sprache der Europäischen Kommission entspricht. Und da die Kommission in offiziellen Statements das Problem der Globalisierung auf die ökonomische Komponente reduziert, beschränken sich die Ausführungen auch auf diesen Bereich der Transnationalisierung. Dieses Vorgehen berücksichtigt die zwei verschiedenen Ebenen der Globalisierungsforschung. Globalisierung ist nicht nur eine bestimmte Form institutionellen Wandels, sondern zugleich auch eine soziale Konstruktion mit politischen Konsequenzen. Für die Sozialwissenschaften ergibt sich daraus der Auftrag, sowohl den unter dem Label „Globalisierung“ stattfindenden institutionellen Wandel selbst zu analysieren als auch zu beobachten, wie relevante Akteure ihrerseits diesen Wandel beobachten und interpretieren. ${ }^{5}$

Als bestimmte Form institutionellen Wandels - und im Unterschied zu früheren Globalisierungsschüben - ist moderne ökonomische Globalisierung die Intensivierung des wirtschaftlichen Austauschs und Wettbewerbs durch die Ausdehnung der Märkte für Güter, Dienstleistungen, Kapital und Arbeit wie auch die dadurch bedingte und als solche wahrgenommene Intensivierung von Interdependenzen zwischen weit voneinander entfernten Lebensumständen. Diese Intensivierungen sind die Folge von Produktionsfaktor-Unterschieden zwischen Volkswirtschaften verschiedener Weltregionen einerseits und von radikal sinkenden Kosten der Überwindung großer räumlicher Distanzen andererseits. ${ }^{6}$ Die ökonomische Globalisierung der Gegenwart vollzieht sich vor allem innerhalb der Triade Nordamerika/Ostasien/Europa, auch wenn sich die Zusammensetzung der jeweils relevanten Volkswirtschaften dieser drei Weltregionen in einigen Fällen gewandelt haben mag. So galten zum Beispiel noch vor zwei bis drei Jahrzehnten Japan und Südkorea als industriell aufstrebende Schwellenländer Ostasiens; heute trifft diese Beschreibung eher auf China und Indien zu, während Japan und Südkorea sich zu führenden Industrienationen mit Produktivitätsindikatoren über und Wohlstandsindikatoren auf europäischem Niveau entwickelt

5 Therborn, G.: Globalization and Inequality. Issues of Conceptualization and Explanation, in: Soziale Welt, 52/4 (2001), 449-476.

6 Siebert, H.: Locational Competition: A Neglected Paradigm in the International Division of Labour, in: World Economy, 29/2 (2006), 137-159. 
haben. ${ }^{7}$ Und der europäische Teil der Triade ist bekanntlich durch die Transformation der ehemaligen sozialistischen Volkswirtschaften deutlich gewachsen.

Auch wenn durch diese Entwicklungen zweifellos die jeweiligen Produktionsfaktor-Unterschiede innerhalb der drei Regionen angestiegen sind, so sind doch nach wie vor die interregionalen Differenzen prägend für grenzüberschreitendes wirtschaftliches Agieren wie auch für die politischen und sozialen Folgen dieses wirtschaftlichen Agierens. Die grundlegenden Charakteristika der drei cluster ändern sich allenfalls inkrementell, ihre Wirtschaftsprofile lassen sich wie folgt kennzeichnen: ${ }^{8}$

- Die nordamerikanischen Volkswirtschaften sind in hohem Maße Dienstleistungsökonomien. Vor allem die USA weisen ein ebenso dauerhaftes wie hohes Außenhandelsdefizit aus. Dass Nordamerika innerhalb der Triade die bedeutendste Import-Region ist, lässt sich zu einem Gutteil auf Produktionsverlagerungen nach Ostasien und auf die abnehmende eigene industrielle Innovationskraft zurückführen. Dem verbreiteten liberalen Grundverständnis ${ }^{9}$ ist es zu verdanken, dass die sozialen Folgen dieser Globalisierungsverluste keine grundstürzenden politischen Krisen auslösen, stattdessen treibt die "globale“ industrielle Verlagerung den Ausbau der nordamerikanischen Volkswirtschaften zu Dienstleistungsökonomien weiter voran. Die damit zwangsläufig einhergehenden starken Einkommensdifferenzen ${ }^{10}$ sind weitgehend akzeptiert. Forderungen nach sozialstaatlichen Kompensationen werden kaum erhoben, der residualen sozialen Absicherung wegen ist die Arbeitslosigkeit gering und die Einkommensungleichheit (Gini) auch nach Steuern und Transfers sehr hoch. ${ }^{11}$ Staatliche Umverteilung zum Zwecke der sozialen Absicherung findet nur begrenzt statt; stattdessen kommt in Nordamerika dem Markt (in Form entweder betrieblicher Sozialpolitik auf dem Arbeitsmarkt oder freiwilliger privater Vorsorge und Absicherung auf dem Versicherungsmarkt) eine im internationalen Vergleich überdurchschnittlich große Bedeutung zu. ${ }^{12}$

7 Ringen, S./Kwŏn, H./Yi, I./Kim, T./Lee, J.: The Korean state and social policy. How South Korea lifted itself from poverty and dictatorship to affluence and democracy, New York, 2011.

8 Vgl. Vobruba, G.: Integration + Erweiterung. Europa im Globalisierungsdilemma, Wien, 2001, 110.

9 Wildavsky, A.: A cultural theory of expenditure growth and (un)balanced budgets, in: Journal of Public Economics 28/3 (1985), 349-357.

10 Iversen, T./Wren, A.: Equality, Employment, and Budgetary Restraint. The Trilemma of the Service Economy, in: World Politics 50/4 (1998), 507-546.

11 Grell, B.: Unemployment Compensation in the United States. Provisions and Institutional Changes since the 1980s. WZB-Discussion paper 2011-202, Berlin, 2011.

12 Adema, W./Ladaique, M.: How Expensive is the Welfare State? Gross and Net Indicators in the OECD Social Expenditure Database. OECD social, employment and migration working papers, No. 92, Paris, 2009, hier 48 . 
- Spiegelbildlich zu Nordamerika verzeichnen die ostasiatischen Volkswirtschaften, insbesondere China, hohe Außenhandelsüberschüsse. Die Exporte sind möglich, weil der Produktionsfaktor Arbeit nahezu unerschöpflich zur Verfügung steht und seine Kosten nur einen Bruchteil der Arbeitskosten in Nordamerika oder Europa ausmachen. Damit nicht verbunden ist ein hohes Humankapitalniveau der Arbeitskräfte. Die Produktivität je Arbeitskraft ist niedrig. Eigene industrielle Innovationskraft entwickelt sich nur langsam und erst in jüngster Zeit, sie ist zudem oft das Resultat vorheriger Imitation oder des Erwerbs außerasiatischen technischen know-hows. ${ }^{13}$ Das exzessive Wirtschaftswachstum der letzten Jahre ${ }^{14}$ basierte vor allem auf Massenproduktion und auf dem Export dieser Güter nach Nordamerika und Europa, dorthin also, wo diese Produkte in aller Regel entwickelt wurden. Die Kombination aus riesigem Arbeitskraft-Angebot, traditionellen und teilweise vormodern anmutenden Gesellschaftsstrukturen, oft gepaart mit nach demokratietheoretischen Maßstäben unterentwickelten politischen Systemen, hat in den Ländern Ostasiens bislang die Entwicklung eines ernstzunehmenden Arbeits(schutz)rechtes ebenso verhindert wie die Entstehung eines Systems sozialer Sicherung, das den europäischen oder auch nur den nordamerikanischen vergleichbar wäre. ${ }^{15}$ Stattdessen sind mikro-solidarische Netzwerke die zentrale Arena der Wohlfahrtsproduktion im Ostasien auch der Gegenwart, sei es in Form von (ruralen) traditionellen Familienverbänden oder in Gestalt (urbaner) nachbarschaftlicher Gemeinschaften. ${ }^{16}$

- Die Volkswirtschaften der Europäischen Union schließlich zeichnen sich durch im Triade-Vergleich hochproduktive und in hohem Maße innovative Industrien aus. Das ist zum einen Ergebnis eines hohen Niveaus wirtschaftlicher und wirtschaftspolitischer Koordinierung: ausgebaute Systeme der Industriellen Beziehungen, der beruflichen Bildung oder der Unternehmenskooperation in Forschung und Entwicklung tragen zur hohen industriellen Produktivität und Innovationskraft der europäischen Volkswirtschaften ebenso bei wie die staatliche Unterstützung und Förderung dieser Koordinationslösungen. ${ }^{17}$ Zum anderen verdanken sich Innovations- und Produktivitätsniveau auch den Handlungsspielräumen, die vom im globalen Maßstab sehr hohen Niveau sozialer Sicherung und der vergleichsweise starken politischen Regulierung der Arbeitsmärkte eröffnet werden. Bei allen Unter-

13 Adams, F. G./Gangnes, B./Shachmurove, Y.: Why is China so Competitive? Measuring and Explaining China's Competitiveness, in: World Economy 29/2 (2006), 95-122.

14 Zur Veranschaulichung: Allein der Anteil Chinas am globalen Bruttoinlandsprodukt wuchs seit Beginn der 1980er Jahre exponentiell um insgesamt mehr als 800 Prozent (1980: 1,2\%; 2011: 8,6\%; Quelle: UNCTAD, UNCTADstat; Basiswerte in US-Dollar zu konstanten Preisen und Wechselkursen (2005)).

15 Parthasarathi, P.: The Great Divergence, in: Past \& Present 176/1 (2002), 275-293.

16 Heberer, T./Göbel, C.: The politics of community building in urban China, London, 2011.

17 Hall, P.A./Soskice, D. (Hg.): Varieties of Capitalism. The Institutional Foundations of Comparative Advantage, Oxford, 2001. 
schieden im Detail: Die zentrale Arena der Wohlfahrtsproduktion ist in Europa der Staat. ${ }^{18}$ Das hohe Sicherungsniveau stand in Europa bislang der Durchsetzung von Dienstleistungsökonomien nordamerikanischen Stils entgegen. Vor allem aber führt das im globalen Vergleich starke wirtschafts-, sozial- und arbeitsmarktpolitische Engagement der Staaten der Europäischen Union zu hohen Arbeitskosten und begünstigt die Abwanderung einfacher industrieller Produktion und ortsunabhängiger Dienstleistungen in Weltregionen mit berechenbaren politischen Bedingungen, aber niedrigeren Faktorkosten - also nach Ostasien.

\section{III. Ökonomische Globalisierung und Sozialpolitik}

Die kurze Gegenüberstellung der drei Wirtschaftsregionen macht sichtbar, wie die jeweiligen institutionellen Bedingungen des Arbeitskräfteeinsatzes sowie das Niveau und der Ort bzw. die Instanz sozialer Sicherung zur Festigung der jeweiligen wirtschaftlichen Besonderheiten beitragen. Problemlos lassen sich die eingangs eingeführten drei Arenen der gesellschaftlichen Integration und die Elemente der ökonomischen Triade einander zuordnen (Abb. 1). Die zentralen Arenen der Wohlfahrtsproduktion sind in Nordamerika der Markt, in Ostasien die Familie oder andere mikro-solidarische Gemeinschaften, und in Europa der Staat.

Bis auf weiteres ist damit die Frage nach dem gegenwärtigen Verhältnis von ökonomischer Globalisierung und Sozialpolitik zwangsläufig eurozentristisch. Nur in Europa gibt es überhaupt ausgebaute Systeme sozialer Sicherung. Nur in Europa besteht also die Möglichkeit der politischen Diskussion über das Verhältnis von ökonomischer Globalisierung und Sozialstaatlichkeit. Dieser Befund markiert den analytischen Übergang zur oben erwähnten interpretativen Perspektive der Globalisierungsforschung: Wie deuten und bewerten relevante Akteure im akademischen und politischen Diskurs mit Blick auf Fragen sozialer Sicherheit die Prozesse gegenwärtiger, moderner ökonomischer Globalisierung? Im Folgenden werden die Argumente dieser Diskussion systematisiert, allerdings

18 Wildavsky, A.: a.a.O. Selbstverständlich kann mit Blick auf die europäischen Sozialstaaten weder von struktureller noch von performanter Gleichheit die Rede sein. Auch innerhalb Europas haben die drei Arenen der Wohlfahrtsproduktion im je nationalen Rahmen unterschiedlich starkes relatives Gewicht (s. hierzu Fehmel, T.: Welfare state convergence in Europe. On the structural approximation of European social security systems, in: European Journal of Transnational Studies 4/2 (2012), 54-80). Insgesamt aber - und namentlich im direkten Vergleich mit Nordamerika und Ostasien - zeichnen sich die europäischen Sozialsysteme durch eine besonders starke und dominante Rolle des Staates aus (vgl. Obinger, H./Wagschal, U.: Families of Nations and Public Policy, in: West European Politics 24/1 (2001), 99-114). 
Abbildung 1: Wohlfahrts- und Integrationsarenen im Triade-Vergleich

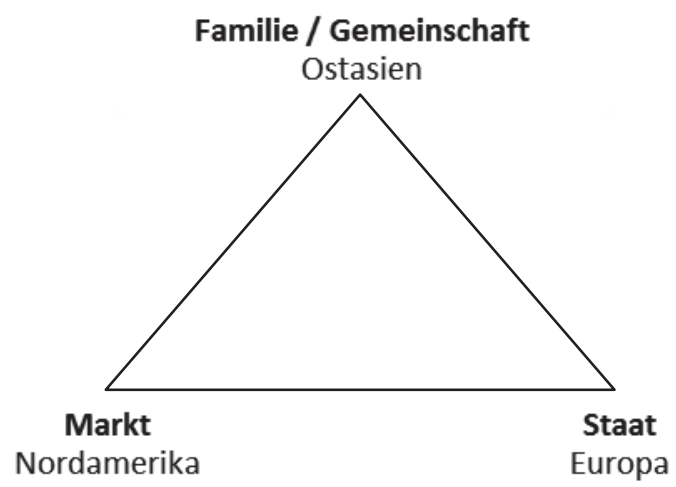

Quelle: eigene Darstellung.

unter Beschränkung auf die grundsätzlich sozialpolitik-affinen Diskussionsteilnehmer.

Drei Lesarten zum Verhältnis von ökonomischer Globalisierung und Sozialpolitik lassen sich unterscheiden. Der ersten, stark pessimistischen Deutung zufolge untergraben die Folgen der ökonomischen Globalisierung die sozialpolitischen Möglichkeiten der ausgebauten europäischen Wohlfahrtstaaten und verhindern den sozialpolitischen Ausbau in den neuen EU-Mitgliedsländern Süd- und Ostmitteleuropas. Die Abwanderung von Kapital und Produktion insbesondere in die aufstrebenden Ökonomien Ostasiens ziehe in den europäischen Volkswirtschaften (und auch in den USA) einerseits eine Verschärfung der Arbeitslosigkeit nach sich und führe andererseits zu sinkenden Staatseinnahmen, weshalb zugleich die Möglichkeiten schwinden, im nationalen Rahmen sozial- und beschäftigungspolitisch auf die Globalisierungsfolgen zu reagieren. Im Bemühen, für Investoren und Produzenten attraktiv zu bleiben (oder wieder zu werden), würden die europäischen Wohlfahrtsstaaten zudem in einen globalen Wettbewerb um möglichst geringe Abgabensätze treten, dadurch aber ihre Probleme bei der Finanzierung ihrer sozialpolitischen Aufgaben lediglich verschärfen. Auch die Löhne würden in einen globalen Unterbietungswettbewerb gezwungen, was freilich nur dazu führe, dass das Nachfragepotential der europäischen und nordamerikanischen Absatzmärkte für die nun verstärkt in Ostasien produzierten Güter massiv schwinde. Dennoch blieben die ostasiatischen Ökonomien mangels eigener Binnennachfrage von den schwächer werdenden Märkten Europas und Nordamerikas abhängig. Eine solche Befürchtung einer Abwärts-Spirale und die entsprechende Deutung der ökonomischen Globalisierung als Negativsummen- 
spiel sind stark verbreitet, empirisch aber nicht haltbar. Von einem wohlfahrtsstaatlichen race to the bottom kann nicht die Rede sein. ${ }^{19}$

Die zweite, moderat pessimistische Lesart betrachtet aus Sicht der europäischen Volkswirtschaften Globalisierung als Nullsummenspiel zugunsten der aufstrebenden Schwellenländer. Sie behält zwar die Ansicht bei, dass die Abwanderung von Kapital und Produktion nach Asien in Europa zu Arbeitsplatzverlusten führt, verabschiedet jedoch die Auffassung sinkender wohlfahrtsstaatlicher Handlungsfähigkeit in Europa infolge sinkender öffentlicher Einnahmen. Von Globalisierungsprozessen betroffen sind nicht ganze Volkswirtschaften. Betroffen sind Unternehmen, zuweilen auch ganze Branchen, und für die dort Beschäftigten mag das durchaus unangenehme Folgen haben. Bislang jedoch haben sich die etablierten Wohlfahrtsstaaten mit ihren jahrzehntelangen StrukturwandelErfahrungen als reaktionsfähig und leistungsstark genug erwiesen, auch die Folgen der ökonomischen Globalisierung zu bewältigen und die mit ihr einhergehenden Beschäftigungs- und Einkommensverluste zu kompensieren. Indem Sozialpolitik die Globalisierungsverlierer für ihre Arbeitsplatzverluste entschädigt, trägt sie zur Akzeptanz des politischen Projektes Globalisierung bei. Und mit sozialpolitischen Leistungen lässt sich die Zeitspanne überbrücken, die die Globalisierungsverlierer benötigen, um sich individuell an die Arbeitsmarkterfordernisse anzupassen, die sich aus globalisierten Wettbewerbsbedingungen ergeben. Diese Lesart der Globalisierung und der sozialpolitischen Beherrschbarkeit ihrer Folgen hat einen wesentlich höheren Realitätsgehalt als die oben dargestellte. Gleichwohl ist sie moderat pessimistisch, denn zu den impliziten Voraussetzungen dieser Deutung gehört es, die ökonomische Globalisierung als negativ und als unter sozialen Gesichtspunkten nachteilig wahrzunehmen und zu kommunizieren. Insofern ist der von der Europäischen Kommission aufgelegte Europäische Fonds für die Anpassung an die Globalisierung (EGF) durchaus heikel. Aus institutionalistischer Perspektive ist das Bemühen der Kommission nachvollziehbar, sich selbst als sozial- und beschäftigungspolitisch problemwahrnehmungs- und handlungsfähigen Akteur darzustellen. Das aber hat die Konsequenz, dass die diffuse negative Globalisierungswahrnehmung der Leute durch solche Programmbezeichnungen verstärkt und nicht etwa abgebaut wird. Vor allem bleibt das Verhältnis von ökonomischer Globalisierung und ökonomischer Europäisierung unklar. Der Globalisierung gesteht die EU durchaus behand-

19 Busemeyer, M.R.: From myth to reality: Globalisation and public spending in OECD countries revisited, in: European Journal of Political Research 48/4 (2009), 455-482. 
Abbildung 2: Drei Lesarten zum Verhältnis von ökonomischer Globalisierung und Sozialpolitik

\begin{tabular}{cccc}
\hline Negativsummenspiel: & Globalisierung & $\rightarrow$ & Sozialpolitik \\
\hline Nullsummenspiel: & Globalisierung & $\rightarrow$ & Sozialpolitik \\
\hline Positivsummenspiel: & Sozialpolitik & $\rightarrow$ & Globalisierung \\
\hline
\end{tabular}

Quelle: eigene Darstellung.

lungswürdige soziale Folgen zu, während sie das europäische BinnenmarktProjekt durchweg als positiv und vorteilhaft präsentiert. In den Augen national orientierter EU-Bürgern hat der europäische Binnenmarkt aber ähnliche Effekte wie die Globalisierung; er wird von ihnen durchaus als wesentlicher Teil und regionale Variante ökonomischer Globalisierung verstanden.

Vergleichsweise jung und noch wenig verbreitet ist eine dritte Lesart, der zufolge ökonomische Globalisierung weder ein Negativ- noch ein Nullsummenspiel zu Lasten Europas, sondern ein Positivsummenspiel ist, von dem auch die europäischen Gesellschaften profitieren oder zumindest profitieren können. Als Grundlage dieser Lesart sei eine transnationale Anwendung des Kuznets-Theorems ${ }^{20}$ vorgeschlagen: Die Annahme dauerhaft stabiler Faktor- und Wohlstandsunterschiede zwischen den drei Wirtschaftsblöcken ist unplausibel. Stattdessen ist, wie die erwähnten Beispiele Japan und Südkorea zeigen, von einer sich zunächst vergrößernden und im weiteren Zeitverlauf wieder verringernden Produktivitätsund Einkommensungleichheit zwischen verschiedenen, miteinander im Austausch stehenden globalen Regionen auszugehen. Immer klarer zeichnet sich ab, dass die exorbitanten Wachstumsraten in Ostasien, namentlich in China und Indien, in den letzten Jahren das Entstehen einer eigenen urbanen Mittelschicht begünstigt haben. Das hat drei für unsere Frage bedeutsame Folgen.

Erstens entwickeln diese Mittelschichten zunehmend politisches Gewicht, das sich aus den mit zunehmendem Wohlstand wachsenden Verlustängsten ergibt und dem sich auch die politische Elite im staatskapitalistisch organisierten China nicht dauerhaft entziehen kann. Durch die Wohlstandszuwächse in der Breite wird Statuserhalt ein politisches Anliegen. Zweitens verstärken der zunehmende 
Umfang und die ansteigende politische Bedeutung der Mittelschichten dem klassischen „nationalökonomischen“ Kuznets-Theorem entsprechend die soziale Ungleichheit im nationalstaatlichen Rahmen. Aus beiden Entwicklungen zusammen ergeben sich politische Impulse: in den letzten Jahren wuchs die Bereitschaft der chinesischen Regierung zu sozialpolitischem Handeln. Am eindrücklichsten zeigt sich das in der Einführung der ersten umfassenden ${ }^{21}$, dem Bismarck-Prinzip folgenden Sozialversicherung in China im Jahr 2011. Die oft beschriebene chinesische Imitationsneigung scheint also neben technologischen zunehmend auch institutionelle Lösungen zu erfassen. Womöglich sind dies die Anfänge einer sozialpolitischen Entwicklung, wie sie im 19. Jahrhundert in (übrigens ebenfalls nicht demokratischen) europäischen Staaten begründet wurde. ${ }^{22}$ Ohne an dieser Stelle auch nur ansatzweise Vorhersagen über mögliche sozialpolitische Entwicklungen in China oder Indien treffen zu können oder zu wollen: es lässt sich absehen, dass sich als Folge der unausweichlichen Befriedigung der steigenden Nachfrage nach staatlicher Umverteilung zum Zwecke des Statuserhalts und der Wiederangleichung der Einkommensunterschiede auch die globalen Produktionsfaktor-Unterschiede reduzieren werden. ${ }^{23}$ Drittens entwickeln die sich ausdehnenden Mittelschichten in diesen Gesellschaften zunehmend selbst ökonomische Nachfrage, die ihrerseits global ausgerichtet ist. Damit entstehen auch für europäische Unternehmen neue Absatzmärkte. ${ }^{24}$ Und in exakt diesem Sinne kann ökonomische Globalisierung ein Positivsummenspiel sein.

Vor diesem Hintergrund wäre es fatal, würde in Europa der Versuch unternommen, durch Sozialkürzungen - und darauf folgende Lohnsenkungen - die Wettbewerbsfähigkeit jener Branchen zu erhalten, die mit niedrig qualifizierter Arbeit mit den ostasiatischen Ökonomien konkurrieren müssen. Der Punkt ist nicht in erster Linie, dass sozialpolitisch destabilisierte, niedrig bezahlte industrielle Beschäftigung in Europa zu unbefriedigenden Lebensbedingungen führt. Entscheidend ist, dass sich durch die aufgrund der Sozialkürzungen verbesserte Wettbewerbsfähigkeit solcher Branchen in Europa zwangsläufig die Beschäftigungs- und Einkom-

21 Umfasst Renten-, Kranken-, Arbeitslosen-, Arbeitsunfall- und Mutterschaftsversicherung

22 Baldwin, P.: The politics of social solidarity. Class bases of the European welfare state: 1875-1975, Cambridge, 1990.

23 Jüngste Entwicklungen unterstreichen dies: „China steht vor einem Wandel. [...] Die Gehälter steigen und Firmen wandern ab. T-Shirts und Turnschuhe lassen sich in Vietnam oder Bangladesch längst viel preiswerter nähen“ (Süddeutsche Zeitung vom 24.8.2012, 21). Dort jedoch ist das ArbeitskräfteAngebot wesentlich begrenzter als in China.

24 Dauth, W./Findeisen, S./Suedekum, J.: The Rise of the East and the Far East: German Labor Markets and Trade Integration. IAB Discussion Paper 16/2012, Nürnberg, 2012. 
menssituationen der entsprechenden Branchen und Arbeitnehmer in Ostasien verschlechtern. Wenn die Annahme einer für Europa wachsenden Relevanz der wachsenden Nachfrage der wachsenden Mittelschichten in China zutrifft, dann würde eine Verschlechterung der dortigen Beschäftigungs- und Einkommenssituationen über kurz oder lang auch auf die Absatzmöglichkeiten europäischer Unternehmen tangieren. Die Kürzung von Sozialleistungen in Europa und die damit bewirkte Absenkung des Reservationslohns in europäischen niedrigproduktiven Industrien schadet also sowohl den dort beschäftigten niedrig-qualifizierten als auch den in anderen Branchen beschäftigten hoch-qualifizierten Arbeitnehmern. ${ }^{25}$

Von europäischen politischen Akteuren verlangt das - im Sinne einer weltgesellschaftlichen Anwendung des Durkheim'schen Theorems sozialer Arbeitsteilung ${ }^{26}$ - die Einsicht in die Notwendigkeit von Spezialisierung und sozialer Arbeitsteilung im grenzüberschreitenden, globalen Maßstab. Zu einem Positivsummenspiel wird die ökonomische Globalisierung nur, wenn es gelingt, die Fähigkeit großer Teile der europäischen Ökonomien zur breit angelegten, qualifikatorisch anspruchsvollen Entwicklung innovativer technisch-industrieller Produkte und wissensintensiver Dienstleistungen aufrecht zu erhalten und zu stärken. An diesem Punkt kommen die spezifischen europäischen Besonderheiten vergleichsweise ausgebauter Systeme sozialer Sicherung einerseits, die entsprechenden Deutungsmuster der Europäischen Kommission andererseits ins Spiel.

\section{IV. Ökonomische Globalisierung und europäische Sozialpolitik}

Der wichtigste Unterschied zwischen der im Jahr 2000 verabschiedeten Lissabon-Strategie der Europäischen Union und der im Jahr 2010 initiierten Nachfolgestrategie „Europa 2020“ ist die weltwirtschaftliche Selbstverortung.

Mit der Lissabon-Strategie hatten sich die Staats- und Regierungschefs der Europäischen Union noch das Ziel gesetzt, binnen zehn Jahren ,die Union zum wettbewerbsfähigsten und dynamischsten wissensbasierten Wirtschaftsraum in der Welt zu machen - einem Wirtschaftsraum, der fähig ist, ein dauerhaftes Wirtschaftswachstum mit mehr und besseren Arbeitsplätzen und einem größeren sozialen Zusammenhalt zu erzielen“. ${ }^{27}$ Zehn Jahre später ist von diesem An-

25 Atkinson, T.: Social Inclusion and the European Union, in: Journal of Common Market Studies 40/4 (2002), 625-643.

26 Durkheim, E.: Über soziale Arbeitsteilung. Studie über die Organisation höherer Gesellschaften. Mit einer Einleitung von Niklas Luhmann. Frankfurt/M., 2008.

27 Europäischer Rat: Schlussfolgerungen des Vorsitzes, Brüssel, 2000. 
spruch keine Rede mehr. Stattdessen wird explizit anerkannt, dass momentan außereuropäische Industrie- und Schwellenländer die treibenden weltwirtschaftlichen Wachstumsmotoren sind und dass die europäischen Ökonomien einen großen Teil ihres Wachstums durch Handel mit den Schwellenländern werden erwirtschaften müssen. ${ }^{28}$ Die EU wird nicht mehr als isolierter Wirtschaftsraum dargestellt, sondern als ,größte Handelsmacht des Globus“, die aus dem Nachfrageboom der aufstrebenden Mittelschichten der Schwellenländer Nutzen ziehen könne. Das ist nicht weniger als eine Relativierung der bisherigen eigenen Position im Weltwirtschaftsgefüge.

Diese Neubewertung globalen ökonomischen Wettbewerbs geht einher mit der Reflektion, dass sich die Europäische Union auf ihre komparativen Vorteile besinnen müsse, um die Herausforderung der zunehmenden globalen Konkurrenz zu bewältigen. In der Vergangenheit lagen diese Spezialisierungsvorteile vor allem in humankapitalintensiver Produktion. Sie bestanden in der Fähigkeit europäischer Unternehmen, Waren und Dienstleistungen zu entwickeln und anzubieten, die in Europa selbst einen hohen technischen und sozialen Standard gewährten und auch außerhalb Europas auf Interesse stießen. Und sie bestanden in der Fähigkeit hochqualifizierter Arbeitnehmer, diese Waren und Dienstleistungen zu produzieren bzw. zu erbringen. Diese Fähigkeiten sind der Einschätzung der Kommission zufolge geschwunden. Folgerichtig ruft sie im Strategiepapier „Europa 2020“ das Ziel einer „Innovationsunion“ aus und entwirft sowohl Leitinitiativen als auch konkrete Maßnahmen, um ,sicherzustellen, dass innovative Ideen in wachstums- und beschäftigungswirksame Produkte und Dienstleistungen umgesetzt werden können“. Diese Zielbestimmung hat sozialpolitische Konsequenzen. Im engeren und weiteren Sinne sozialpolitisch relevant für die Innovationsunion sind hierbei die Leit-Themen Bildung einerseits sowie (im Zusammenhang mit der Leitinitiative Beschäftigung und Qualifikationen) das Ziel der Modernisierung der sozialen Sicherung andererseits. ${ }^{29}$ Auf das erstgenannte Thema sei nachfolgend nur kurz, auf das zweite etwas ausführlicher eingegangen.

28 Europäische Kommission: Europa 2020, a.a.O, 27.

29 Im hier diskutierten Kontext ist die Leitlinie „Europäische Plattform zur Bekämpfung der Armut“ innerhalb des Strategiepapiers „Europa 2020“ sozialpolitisch nicht relevant. Sie lässt einen Zusammenhang zum Ziel der Innovationsunion kaum erkennen. Sie steht nicht nur inhaltlich quer, indem sie ohne jede Kausalverknüpfung auskommt, die allen anderen Leitlinien mit Blick auf Wachstum und Wettbewerbsfähigkeit zu eigen ist. Sie erscheint auch in beachtlicher Weise und anders als alle anderen Zielbestimmungen vage und unterbestimmt. Man kommt nicht umhin, die Aufnahme des Ziels der Armutsbekämpfung in die Strategie als symbolisches Zugeständnis zu betrachten, mit dem der ansonsten sehr ökonomie-lastige Zukunftsentwurf „Europa 2020“ in Balance gebracht werden soll. 


\section{Innovation und Bildung}

Innovationen entstehen nicht aus dem Nichts. Sie vollziehen sich in längeren Zeiträumen. Selbst geniale, pfadbrechende Einfälle bedürfen zumindest der anschließenden strategischen Entwicklung, um sich nachhaltig auf Märkten durchsetzen zu können. Erfolgreiche (auch radikale) Innovationen entwickeln sich also üblicherweise inkrementell, haben eine mehr oder minder ausgedehnte Vorlaufzeit und bedürfen innovationsfähiger Unternehmer und Beschäftigter. ${ }^{30}$ Aber auch Innovationsfähigkeit ergibt sich nicht aus dem Nichts. Sie erfordert in der Regel den vorherigen Erwerb von Wissen und den Aufbau von Spezialisierung. Die politische Leitlinie der Europäischen Kommission, selbst verstärkt in Bildung und Bildungsinfrastruktur zu investieren wie auch die Mitgliedstaaten dazu anzuhalten, ist insofern nicht falsch, aber auch nicht originell. Die Leitlinie schließt an eine Vielzahl früherer Zielbestimmungen und Maßnahmen der EU an. ${ }^{31}$ Sie folgt unverändert - und unverändert explizit - der verbreiteten Annahme, dass mit intensivierten Bildungsanstrengungen verstärkt Innovationsfähigkeit generiert werden kann, die sich in Innovationen niederschlägt, deren Marktfähigkeit für unternehmerischen Erfolg sorgt, von dem wiederum allgemeine Wachstumsimpulse ausgehen, die zu Wohlstandsgewinnen führen. ${ }^{32}$ Hier soll weder die Angemessenheit dieser Kausalitätsannahme diskutiert ${ }^{33}$ noch die daran geübte Kritik einer ökonomistischen Engführung dieses Ansatzes auf employability als Bildungsziel vertieft werden. ${ }^{34}$ Denn dem Grunde nach sind bildungspolitische Aspekte nicht Teil sozialpolitischer Erörterungen; jedenfalls dann nicht, wenn man soziale Sicherung im Anschluss an Ganßmann definiert als den Erwerb und die Einlösung von Ansprüchen an andere für den Fall, dass man sich

30 Castellacci, F.: Technological paradigms, regimes and trajectories: Manufacturing and service industries in a new taxonomy of sectoral patterns of innovation, in: Research Policy 37/6-7 (2008), 978-994.

31 Z.B. Europäische Kommission: Lehren und Lernen - Auf dem Weg zur kognitiven Gesellschaft. Weißbuch zur allgemeinen und beruflichen Bildung. Luxemburg (KOM(95) 590 endg), 1995; Europäische Kommission: Einen europäischen Raum des lebenslangen Lernens schaffen. Mitteilung der Kommission. Brüssel (KOM(2001) 678 endg), 2001; Europäische Kommission: Wissen, Kreativität und Innovation durch lebenslanges Lernen. Mitteilung der Kommission. Brüssel (KOM(2007) 703 endg), 2007.

32 Exemplarisch für viele: Merkel, W.: Soziale Gerechtigkeit und die drei Welten des Wohlfahrtskapitalismus, in: Berliner Journal für Soziologie 11/2 (2001), 135-157.

33 Dazu Nguyen, T./Pfleiderer, M.: Welchen Einfluss hat das Humankapital auf das Wirtschaftswachstum?, in: Jahrbuch für Wirtschaftswissenschaften 63/1 (2012), 68-83.

34 Dazu Koch, G.: Reform des Bildungswesens: Wissensbasierte Gesellschaft und Globalisierung, in: Linzbach, C./Lübking, U./Scholz, S./Schulte, B. (Hg.): Globalisierung und europäisches Sozialmodell, Baden-Baden, 2007, 235-254. 
nicht (mehr) durch eigene Leistung versorgen kann. ${ }^{35}$ Dass man die genannten bildungspolitischen Positionen der Europäischen Kommission dennoch bei der Beschäftigung mit Problemen sozialer Sicherung nicht vollends außer Acht lassen sollte, ist der Tatsache geschuldet, dass im politischen Diskurs in zunehmendem Maße Bildungspolitik als präventive Sozialpolitik thematisiert wird. ${ }^{36}$ Die sich daraus ergebende Frage nach dem zukünftigen Verhältnis von präventiver und nachsorgender Sozialpolitik ist durchaus von Belang, aber sie wird hier nicht weiter diskutiert. ${ }^{37}$ Interessanter ist, dass die Europäische Kommission das Thema Bildung in einer Weise und mit Sprachmustern einführt, die darauf schließen lassen, dass sie Bildung und ihre Bedeutung für Wohlstand und soziale Kohäsion als Substitut für die ausbleibenden Erträge ihrer Kohäsionsanstrengungen betrachtet. In Ermangelung einer eigenständigen europäischen Sozialpolitik und angesichts der in der Gegenwart nur wenig erfolgreichen europäischen Strukturpolitik verspricht die Betonung von Bildung zumindest Wohlstandsgewinne in der Zukunft. Die Verlagerung politischer Ansprüche in die Zukunft kann der Reduzierung von Legitimitätsrisiken in der Gegenwart dienen. Dass das Thema Bildung (jenseits eines europäisierten Hochschulraums) für europäische Regulierung und Koordinierung geeignet ist, muss mit Blick auf die Unterschiede der nationalen Bildungssysteme und auf die Präferenzen der Europäer allerdings bezweifelt werden: Neben der Sozial- ist die Bildungspolitik der Politikbereich, für den die Befragten am nachdrücklichsten eine nationale Gestaltungshoheit präferieren. ${ }^{38}$

\section{Innovation und soziale Sicherung}

Innovatives Handeln ist riskant; die Risiken ergeben sich aus seinem experimentellen Charakter. Sie erwachsen mithin aus der Ungewissheit der Zukunft; aus unzureichenden Informationen darüber, ob die gewählten Wege zum angestrebten Ziel führen und die eingegangenen Investitionen den erwarteten Ertrag bringen werden. Bekannt sind lediglich die Kosten einer Innovation. Es gehört zum

35 Ganßmann, H.: Soziale Sicherheit durch die EU? Staatstheoretische und europasoziologische Perspektiven, in: Eigmüller, M./Mau, S. (Hg.): Gesellschaftstheorie und Europapolitik, Wiesbaden, 2010, 329352.

36 Vgl. Randhahn, S.: Bildungspolitik im deutschen Sozialstaat. Die soziale Dimension bildungspolitischer Programme und Prozesse in vier Bundesländern. Baden-Baden, 2011.

37 S. dazu Allmendinger, J./Nikolai, R.: Bildungs- und Sozialpolitik. Die zwei Seiten des Sozialstaats im internationalen Vergleich, in: Soziale Welt 61/2 (2010), 105-119.

38 Fehmel, T.: Sicherungsbewahrung. Europas sozialpolitische Zukunft, Weinheim, Beltz Juventa, 2013. 
sozialpsychologischen wie zum organisationssoziologischen Basiswissen, dass Akteure sich in einer Entscheidungssituation, die geprägt ist durch sichere Kosten, aber ungewisse Erträge, nur ungern riskant verhalten und sich stattdessen an bestehende Erfahrungen und eingespielte Verhaltensmuster halten. Innovatives Handeln widerspricht dem grundsätzlichen Sicherheitsbedürfnis von Menschen und Unternehmen, von Branchen und ganzen Volkswirtschaften. ${ }^{39}$ Das gilt selbst dann, wenn die Verweigerung innovativen Handelns erkennbar dazu führt, dass eine Person, ein Unternehmen, eine Branche oder Volkswirtschaft einen (durch früheres innovatives Handeln) erworbenen Status einbüßt - sei es im lokalen oder im globalen, im ökonomischen oder im außerökonomischen Maßstab. ${ }^{40}$

Daraus ergibt sich die Herausforderung, innovatives Handeln zu ermöglichen, indem die Stabilitäts- und Sicherungsinteressen der beteiligten Akteure gewahrt werden. Das ist eine politische Aufgabe - bezogen auf Branchen und Unternehmen eine wirtschafts- und forschungspolitische, bezogen auf Personen und Interessengruppen in erster Linie eine sozialpolitische. ${ }^{41}$ Im Triade-Vergleich haben die europäischen Systeme sozialer Sicherung das größte Potential, den Widerspruch von Innovationsnotwendigkeit und Sicherheitsbedürfnis in eine produktive Kombination aus innovativem Handeln und sozialer Sicherheit umzuformen. Wenn also Sicherheit ein Generalschlüssel für Flexibilität, Kreativität und Innovationsstreben ist, und all dies wiederum die Voraussetzung für ökonomische Effizienzsteigerungen im modernen Kapitalismus, ${ }^{42}$ dann reagiert Sozialpolitik nicht primär auf ökonomische Globalisierung, sondern ermöglicht sie.

\section{V. „Europa 2020“ und soziale Sicherung}

In ihrem Strategiepapier „Europa 2020“ hält die Europäische Kommission die Modernisierung der sozialen Sicherung als zentrales Ziel fest. Sie versteht unter dieser Modernisierung explizit die „Umsetzung der Flexicurity-Grundsätze [zum Zwecke] ... der Befähigung der Menschen, sich mittels der Aneignung neuer

39 Zapf, W.: Individualisierung und Sicherheit. Einige Anmerkungen aus soziologischer Sicht, in: Rolf, G/ Spahn, P.B./ Wagner G.G. (Hg.): Sozialvertrag und Sicherung. Zur ökonomischen Theorie staatlicher Versicherungs- und Umverteilungssysteme, Frankfurt/M., 1988, 371-380.

40 Olson, M.L.: The rise and decline of nations. Economic growth, stagflation, and social rigidities, New Haven, 1982.

41 Vgl. dazu Heidenreich, M.: Innovationen und soziale Sicherung im internationalen Vergleich, in: Soziale Welt 55/2 (2004), 125-144.

42 Vobruba, G.: Die Gesellschaft der Leute. Kritik und Gestaltung der sozialen Verhältnisse, Wiesbaden, $2009,98$. 
Qualifikationen an neue Gegebenheiten anzupassen und sich beruflich neu zu orientieren" ${ }^{43}$ Sie hält also vor dem Hintergrund des Innovations-Imperativs sowohl den lebenslangen Erwerb von arbeitsmarktrelevanten Qualifikationen als auch die Bereitschaft der Erwerbstätigen zu Mobilität, also zum Wechsel zwischen zwei Arbeitsverhältnissen, vor allem aber in temporären Arbeitslosigkeitsphasen, für unverzichtbar.

Zumindest explizit verbinden weder die Kommission selbst noch die von ihr eingesetzte Forschungsgruppe zur Operationalisierung des Flexicurity-Ansatzes $^{44}$ mit dieser Vorstellung eine bestimmte Form der Organisation sozialer Sicherungssysteme. ${ }^{45}$ Trotz der zentralen Zielbestimmung der Modernisierung der sozialen Sicherung bleiben konkretisierende Aussagen der Kommission im Kontext der Strategie „Europa 2020“ selten. Mit Blick auf die Alterssicherung (a) ist sehr allgemein von Renten die Rede, die erträgliche Lebensbedingungen und Unabhängigkeit ermöglichen sollen. Allerdings wird auf Reformen in einigen Ländern hingewiesen, mit denen die Verbindung zwischen Beitrag und Rentenhöhe gestärkt wird. Auch wird die (weitere) Aufwertung der zweiten und dritten Säule der Altersvorsorge gefordert, was sich als Präferenz für eine beitragsfinanzierte Alterssicherung lesen lässt. Andererseits wird dringend empfohlen, die Lohnnebenkosten (zu denen explizit auch Sozialversicherungsbeiträge gehören) zu senken, um insbesondere im Niedriglohnbereich die Anreize für Arbeitsplatzschaffung und Arbeitsaufnahme zu erhöhen. Im Gegenzug wird angeregt, die sich daraus ergebenden Einnahmeausfälle durch indirekte Steuern und Konsumsteuern zu kompensieren (b). „Über-generösen“ Arbeitslosenunterstützungen lässt sich der Kommission zufolge wirksam mit Instrumenten aktiver Arbeitsmarktpolitik, insbesondere mit Aktivierungsstrategien begegnen (c). Ab welcher Höhe und unter welchen sonstigen Bedingungen (Bezugsdauer, Anwartschaftszeit etc.) Arbeitslosenunterstützung als ,übergenerös“ zu gelten hat, bleibt allerdings unklar, obwohl eine Vielzahl möglicher Indikatoren aufgelistet und ein benchmarking angeregt wird (d). ${ }^{46}$

43 Europäische Kommission: Europa 2020, a.a.O., 22.

44 Manca, A. R./Governatori, M./Mascherini, M.: Towards a set of Composite Indicators on Flexicurity. A Comprehensive Approach. Luxemburg: Publications Office of the European Union. European Commission, Joint Research Centre, Institute for the Protection and Security of the Citizen, 2010.

45 Fehmel, T: Interessen und Intentionen. Konvergenzeffekte und Realisierungschancen des FlexicurityKonzepts der EU, in: Zeitschrift für Staats- und Europawissenschaften 9/2 (2011), 199-223.

46 Quellen einsehbar unter http://ec.europa.eu/europe2020/. 
Ungeachtet dieser Unbestimmtheit lässt sich allerdings zeigen, dass die von der Kommission im Strategiepapier miteinander verbundenen Themen Globalisierung und Aufstieg der Schwellenländer einerseits, Innovationsnotwendigkeit und arbeitsmarktrelevante Qualifikation im Berufsleben (also jenseits des Bildungssystems) in Europa andererseits implizit ein bestimmtes Gestaltungsmuster sozialer Sicherung begünstigen. Dafür sei auf die oben angestellten Überlegungen zum Zusammenhang von Innovation und Spezialisierung sowie zum Widerspruch von Innovation und Sicherheit zurückgegriffen, unter Ergänzung um interessentheoretische Argumente, die den wirtschaftlichen Wert von Sozialpolitik in den Mittelpunkt rücken.

Ausgangspunkt sind jüngere Befunde der Wohlfahrtsstaatsforschung, die zu einer Revision der klassischen Machtressourcen-Theorie und ihrer Grundannahme eines starken Klassenantagonismus zwischen „Kapital“ und „Arbeit“ zwingen. Anders als diese Theorie annimmt, verdanken sich sozialpolitische Entwicklungen keineswegs nur dem Drängen nationaler Arbeiterbewegungen gegen den prinzipiellen Verhinderungswillen der Arbeitgeber. Vielmehr haben auch bestimmte Gruppen von Arbeitgebern ein deutliches Eigeninteresse an Sozialpolitik. Dafür lassen sich im Wesentlichen zwei Motive ausmachen: Mit sozialstaatlichen Arrangements können einerseits branchenübergreifend die Kosten verteilt werden, die mit den besonders hohen sozialen Risiken der Beschäftigten in stark gesundheitsgefährdenden oder arbeitsplatzgefährdeten Branchen verbunden sind, von den Unternehmen dieser Branchen allein aber weder getragen noch abgewiesen werden können. Und sozialstaatliche Instrumente spielen andererseits für die Beschäftigungsstrategien jener Unternehmen eine bedeutsame Rolle, die auf ein stabil hohes Qualifikationsniveau ihrer Beschäftigten angewiesen sind. Dem liegt folgende Überlegung zugrunde: Von Investitionen in Qualifikation können sowohl Unternehmen als auch Arbeitnehmer profitieren. Für die Unternehmen zahlt sich der Wissenszuwachs ihrer Mitarbeiter in höherer Produktivität aus, Arbeitnehmer können durch Qualifizierungen und Spezialisierung höhere Einkommen erzielen. Diesem betriebswirtschaftlichen Basis-Theorem stehen reale Schwierigkeiten gegenüber, die sämtlich in der Einsicht kulminieren, dass das Erreichen der durch Qualifizierung angestrebten Ziele (Produktivitätssteigerung, Lohnzuwächse) keineswegs sicher ist. Investitionen in Wissen sind riskant für Arbeitnehmer und Unternehmen. Sie können sich zum einen als Fehlinvestitionen erweisen, als Investition in Wissen, das nicht nachgefragt wird. Qualifikation und Spezialisierung können zum anderen zwar den Wert der Arbeitskraft in einem bestimmten Wissensgebiet erhöhen, sie grenzen jedoch damit zugleich in 
der Regel auch den Bereich ein, in dem sie auf unternehmerische Nachfrage stoßen. Investitionen in Wissen sind also inhaltliche Selbstbindungen und zudem langfristig wirkende Selbstbeschränkungen, gehen also immer auch einher mit Opportunitätskosten. ${ }^{47} \mathrm{Um}$ sich auf Qualifizierungen und Spezialisierungen einzulassen, benötigen daher insbesondere die Arbeitnehmer Garantien, dass bei einem Lohnausfall die zu erwartende Lohnersatzleistung immer noch über dem Lohnniveau für unqualifizierte bzw. weniger spezialisierte Arbeit liegt: Die Aussicht auf statussichernde lohnproportionale Lohnersatzleistungen stärkt bei Arbeitnehmern die Bereitschaft zu Investitionen in ihr Humankapital und in die Verwertbarkeit ihrer Arbeitskraft.

Kalkulierbare Lohnausfälle können nur solche sein, die sich aus der Tätigkeit selbst ergeben oder aus dem wirtschaftlichen Bereich, in dem sie ausgeübt wird. Tätigkeitsbezogene Ausfallrisiken ergeben sich üblicherweise aus Gesundheitsgefährdungen des entsprechenden Berufes. Branchenbezogene Ausfallrisiken bestehen in Konstellationen starken Wettbewerbs und dynamischer technologischer Entwicklung. In diesen Fällen besteht das Ausfallrisiko nicht im Verlust der individuellen Arbeitsfähigkeit, sondern im Verlust von Arbeitsplätzen.

Im Kontext der ökonomischen Globalisierung ist letzteres der interessantere Fall: Es geht um den Bedarf an statussichernder sozialer Absicherung bei Arbeitslosigkeit. Diesem Interesse hochspezialisierter, branchen- oder betriebsspezifisch qualifizierter Arbeitnehmer trägt ein Sozialtransfersystem mit Beitrags-/Leistungsäquivalenz stärker Rechnung als bedürftigkeitsabhängige universalistische Sicherheitslösungen. Und Unternehmen, die ihrerseits auf die Qualifizierungsbereitschaft ihrer Beschäftigten angewiesen sind, dürften mit dem Hintergrundwissen der Existenz eines derartigen Systems der sozialen Absicherung des Einkommensausfalls bei Arbeitsplatzverlust eher bereit sein, sich im betrieblichen Binnenverhältnis an der Investition der Arbeitnehmer in Qualifikation und Spezialisierung zu beteiligen.

Entsprechend erfasst eine solche Präferenzstruktur nicht unterschiedslos alle Branchen und Arbeitgeber (vgl. Abb. 3). Sozialtransfersysteme mit Beitrags- und/oder Leistungsäquivalenz werden vor allem präferiert von Unternehmen mit Arbeitsplätzen, die a) ein dauerhaft hohes individuelles Qualifikationsniveau erfordern

47 Streeck, $W$.: Skills and the Limits of Neo-Liberalism: The Enterprise of the Future as a Place of Learning, in: Work, Employment \& Society 3/1 (1989), 89-104. 
Abbildung 3: Präferenzstruktur bei Arbeitgebern bzgl. der Gestaltung sozialer Sicherung

\begin{tabular}{|c|c|c|c|}
\hline & \multicolumn{2}{|c|}{$\begin{array}{l}\text { Spezialisierungs- und Qualifikations- } \\
\text { niveau der Beschäftigten }\end{array}$} \\
\hline & & niedrig & hoch \\
\hline \multirow{2}{*}{ 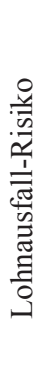 } & 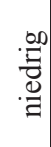 & $\begin{array}{l}\text { kein Absicherungs- } \\
\text { bedarf, weil keine } \\
\text { Investition }\end{array}$ & $\begin{array}{l}\text { kein Absicherungs- } \\
\text { bedarf, weil Investiti- } \\
\text { onsertrag vermeintlich } \\
\text { garantiert }\end{array}$ \\
\hline & 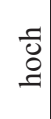 & $\begin{array}{l}\text { bedürftigkeits- } \\
\text { abhängige, universa- } \\
\text { listische Absicherung }\end{array}$ & $\begin{array}{c}\text { Sozialversicherung } \\
\text { mit Einkommens-/ } \\
\text { Leistungsäquivalenz }\end{array}$ \\
\hline
\end{tabular}

Quelle: eigene Darstellung.

und b) einem relativ hohen Lohnausfall-Risiko unterliegen, gegen das es sich sozialpolitisch abzusichern gilt. ${ }^{48}$

Auch im Außenverhältnis, d.h. im politischen Raum und dort kommuniziert über ihre Verbände, bevorzugen, befürworten und verteidigen gegebenenfalls auch die Unternehmen mit dauerhaft hohem Humankapitalbedarf und zugleich hohem Ausfall-Risiko ein System sozialer Sicherung, dass einerseits dem Äquivalenzprinzip folgt, andererseits die damit verbundenen Kosten möglichst weit streut. Unterstützt werden sie von den Industriegewerkschaften, also den Interessenvertretern der Facharbeiterschaft in weltmarktabhängigen Branchen. In diesem Punkt gibt es in Europa übrigens kaum Unterschiede zwischen Sozialsystemen Bismarckscher Prägung und den Arbeitslosigkeitsversicherungen universalistischer Wohlfahrtsstaaten: In Dänemark oder Schweden werden die Lohnersatzleistungen bei Arbeitslosigkeit zwar überwiegend aus Steuermitteln finanziert, sie sind aber Äquivalenzbeträge mit vorherigem Lohn als Bezugsbasis. ${ }^{49}$ Hinge-

48 Mares, I.: Warum die Wirtschaft den Sozialstaat braucht. Ein historischer Ländervergleich, Frankfurt/M., 2004, 50.

49 Clasen, J./Viebrock, E.: Das Genter System der Arbeitslosenversicherung - immer noch gewerkschaftliches Rekrutierungsinstrument oder sozialpolitisches Auslaufmodell? Dänemark und Schweden im Vergleich, in: Zeitschrift für Sozialreform 52/3 (2006), 351-371. Lediglich Großbritannien bildet hier mit alters- und bedürftigkeitsabhängig gestaffelten Pauschal-Zahlbeträgen eine Ausnahme. 
gen ist die staatliche Arbeitslosenunterstützung in den Vereinigten Staaten nicht nur sehr niedrig, sondern auch nur begrenzt auf das zuvor erzielte Erwerbseinkommen bezogen. ${ }^{50}$ Das spricht dafür, die erfolgreiche Bewältigung der sozialpolitischen Folgen der ökonomischen Globalisierung nicht nur auf die Größe und Weltmarktoffenheit einer Volkswirtschaft ${ }^{51}$ oder das generelle Niveau ihrer Sozialausgaben $^{52}$ zurückzuführen, sondern auch auf die Art und Weise, wie in ihr das Risiko Arbeitslosigkeit abgesichert wird.

Unter den gegenwärtigen und wohl auch zukünftigen Bedingungen auf den Arbeitsmärkten dürfte das Gros der Unternehmen in Europa weder in der Lage noch willens sein, Arbeitsplätze anzubieten, die mit unbefristeter Beschäftigungsgarantie ausgestattet und vor jeglichem Rationalisierungs- und Verdrängungsrisiko geschützt sind. Selbst vermeintlich sichere Beschäftigungsverhältnisse im öffentlichen Sektor stehen spätestens seit der Staatsschuldenkrise einiger europäischer Staaten unter Druck. Die Folgen der ökonomischen Globalisierung verschärfen diese Situation möglicherweise noch. ${ }^{53}$ Und ohnehin zählt es zu den Standard-Strategien von Arbeitgebern, dauerhafte Beschäftigungs- und Nachfragegarantien ganz grundsätzlich zu vermeiden, wofür wiederum nicht zuletzt „die Globalisierung“ als Begründung dient. ${ }^{54}$ Gleichviel also, ob das Risiko von Arbeitsplatzverlusten und Lohnausfällen nun real ist oder von Arbeitgeberseite lediglich heraufbeschworen wird, es führt in der Heuristik der Abbildung 3 zu einer Betonung der beiden unteren, und hier vor allem des rechten Quadranten, also zur Betonung der Sozialversicherung gegen Arbeitslosigkeit mit Einkommens-/Leistungsäquivalenz. Ruft man sich die eingangs gegenübergestellten Eigenheiten der nordamerikanischen, der ostasiatischen und der europäischen Wirtschaft-Wohlfahrt-Arrangements in Erinnerung, dann lässt sich die Heuristik auf die Triade der ökonomischen Globalisierung übertragen.

Die Verknüpfung der Themen ökonomische Globalisierung, Innovationsnotwendigkeit, Qualifikation und soziale Sicherung im Strategiepapier „Europa 2020“ der Europäischen Kommission lässt sich daher wie folgt paraphrasieren: Unter den Bedingungen globalen Wettbewerbs und den damit einhergehenden hohen

50 Grell, B., a.a.O.

51 Katzenstein, P.J.: Small states in world markets. Industrial policy in Europe, Ithaca, 1985.

52 Jensen, $C$.: Catching up by transition: globalization as a generator of convergence in social spending, in: Journal of European Public Policy 18/1 (2011), 106-121.

53 Ganßmann, H.: Politische Ökonomie des Sozialstaates, Münster, 2000.

54 Bonoli, G./George, V./Taylor-Gooby, P.: European welfare futures. Towards a theory of retrenchment, Cambridge, 2000, 49ff. 
Abbildung 4: Betroffenheitsstruktur der Wirtschaftsregionen

\begin{tabular}{|c|c|c|c|}
\hline & \multicolumn{2}{|c|}{$\begin{array}{l}\text { Spezialisierungs- und Qualifikations- } \\
\text { niveau der Beschäftigten }\end{array}$} \\
\hline & & Niedrig & Hoch \\
\hline 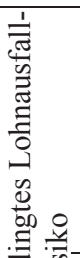 & 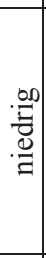 & Ostasien & \\
\hline 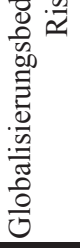 & \begin{tabular}{c} 
\\
\\
0 \\
0 \\
\hdashline \\
\end{tabular} & Nordamerika & Europa \\
\hline
\end{tabular}

Quelle: eigene Darstellung.

und steigenden Risiken von Arbeitsplatzverlusten sind Investitionen in Humankapital die zentrale Möglichkeit, wettbewerbsfähig zu bleiben bzw. zu werden. Von Arbeitnehmern und Unternehmen werden hohe Investitionen in Wissen erwartet - bei hohem Risiko von Fehlinvestitionen. Ein modernes System sozialer Sicherung muss diesem Widerspruch Rechnung tragen; ihm kommt die Aufgabe zu, sozialpolitisch die geforderten Investitionsentscheidungen zu flankieren, die ansonsten womöglich unterbleiben würden. Ein dem Sozialversicherungsprinzip folgendes System mit Beitrags- oder zumindest Einkommens-/Leistungsäquivalenz wird diesem Anspruch - und damit dem Anspruch einer „Innovationsunion" - eher gerecht als ein System mit bedürftigkeitsabhängigen, universalistischen Sozialtransfers.

\section{Schluss}

Emanzipatorisch inspirierte Sozialpolitik ist das nicht. Was sich sozialpolitisch aus dem Innovations-Imperativ der Europäischen Kommission ergibt, dient nicht der Schaffung von Freiräumen jenseits des Arbeitsmarktes bzw. des Erwerbslebens. Es dient vielmehr gerade der Hinlenkung zur Erwerbsorientierung und der Verhaltenssteuerung im Rahmen des Arbeitsmarktes. Ziel dieser sozialen Absicherung ist nicht De-Kommodifizierung im klassisch verstandenen Sinne, sondern Aktivierung zur employability als Instrument der Bewältigung der ökonomischen Globalisierung. Etwas anderes von der EU zu erwarten, von jener 
politischen Ebene, die mit der Durchsetzung der vier Grundfreiheiten das Handeln auf Märkten ins Zentrum ihrer Anstrengungen gestellt hat, wäre naiv.

Aber die wirtschaftlichen Zwecke sozialpolitischer Innovationsförderung determinieren das Handeln der Arbeitnehmer nicht, sie strukturieren allenfalls den Pool ihrer Handlungsoptionen. Im Sinne doppelter Kontingenz entbehren die in einer bestimmten Form von Sozialpolitik zum Ausdruck kommenden Steuerungsbemühungen gestaltungsmächtiger Akteure der Erfolgsgewissheit: Sie müssen mit den Handlungen und Handlungsfolgen auf Seiten der Sozialversicherten keineswegs identisch sein. Denn die Adressaten sozialpolitischer Bemühungen der Verhaltenssteuerung können den mit den sozialpolitischen Instrumenten implizit und explizit verbundenen Erwartungen folgen, müssen es aber nicht. Sie können die im Rahmen von Sozialversicherungen abgegebenen Sicherungsgarantien als Impuls für (riskante) Investitionen in ihr verwertbares Wissen und in ihre Innovationsfähigkeit nutzen. Genauso gut können sie aber auch Sozialversicherungen als Instrumente der Stabilisierung ihres Status quo im „Versicherungsfall“ betrachten und daraus keinerlei arbeitsmarktbezogene Anpassungschancen oder -erfordernisse ableiten.

Diese Interpretationsfreiheit der Versicherten erhöht den Grad ihrer individuellen Freiheit auch jenseits des Arbeitsmarktes. Damit zeigen sich auch in dieser ökonomistisch motivierten Form sozialer Sicherung Unbestimmtheitslücken, ${ }^{55}$ mithin emanzipatorische Anteile. Aus Sicht europäischer politischer Akteure dürfte dies ein nicht-intendierter, aber hinnehmbarer Effekt sein. Denn es ermöglicht den Versicherten, zwischen beiden Interpretationen der Sicherungsgarantien durch Sozialversicherungen - Investitionsanreiz oder Statusabsicherung - zu changieren, sich situativ mal die eine, mal die andere Deutung zu eigen zu machen, und damit zugleich im Rahmen der jeweils gegebenen institutionellen Möglichkeiten zwischen Teilnahme und Nichtteilnahme zu wechseln. Genau diese Interpretationsoffenheit, diese Möglichkeit, ein und dasselbe sozialpolitische Instrument individuell für unterschiedliche Anliegen nutzen zu können, erschwert die Sanktionierung der Nichterfüllung der politischen Erwartungen. Aller Erfahrung zufolge besteht diese Sanktionierung gerade im Ausschluss aus dem Sozialversicherungsprinzip und in der Überführung des „Versicherungsfalles" in steuerfinanzierte Sicherungsformen, denen ein um vieles stärkeres Potential der Verhaltenssteuerung, Aktivierung und inhaltlichen Ausrichtung zu eigen 
ist. Demgegenüber enthält das Prinzip Sozialversicherung einen konstitutiven emanzipatorischen Kern, den es auch über den Kreis der gegenwärtig versicherten abhängig Beschäftigten hinaus fruchtbar zu machen gilt. 